\title{
Triggers of thyroid cancer diagnosis: a systematic review and meta- analysis
}

\author{
Eddy Lincango-Naranjo $\mathbb{D}^{1,2,3}$. Paola Solis-Pazmino $\mathbb{D}^{1,2,3,4}$. Omar El Kawkgi $\mathbb{D}^{1,5}$ - Jorge Salazar-Vega $\mathbb{D}^{3,6}$. \\ Cristhian Garcia $^{3,7} \cdot$ Tannya Ledesma ${ }^{2,3}$. Tatiana Rojas ${ }^{3,8}$ - Benjamin Alvarado-Mafla $\mathbb{1}^{3} \cdot$ Geoffrey Young $^{9}$. \\ Benzon Dy ${ }^{10} \cdot$ Oscar J. Ponce $\mathbb{1}^{1,3,11} \cdot$ Juan P. Brito $\mathbb{B}^{1,3,5}$
}

Received: 28 September 2020 / Accepted: 9 December 2020 / Published online: 29 January 2021

(c) The Author(s), under exclusive licence to Springer Science+Business Media, LLC part of Springer Nature 2021

\begin{abstract}
Purpose Understanding the method of thyroid cancer detection has potential implications on interpreting incidence rates, the diagnosis and management of thyroid cancer. We conducted a systematic review of studies reporting methods of thyroid cancer detection to estimate the frequency of incidentally found cancers and classify triggers of incidental thyroid cancer diagnosis.

Methods We searched multiple bibliographic databases from inception to June 2020. A pair of reviewers, working independently and in duplicate selected studies for inclusion, extracted data, and evaluated each trial's risk of bias. Studies enrolling patients older than 18 years with thyroid cancer confirmed histologically were included.

Results In total, 17 cohorts and 1 cross-sectional study, conducted between 1991 and 2018, enrolling 4668 patients with thyroid cancer were included: $88 \%$ had papillary thyroid cancer and $23 \%$ had papillary thyroid microcarcinoma. The proportion of patients with non-incidental and incidental thyroid cancer was similar: 49\% [95\% confidence interval (CI): 40-58\%]. Subgroup analysis showed that most patients with incidental thyroid cancers had tumor size $<10 \mathrm{~mm}(76 \%$; $95 \%$ CI: 56-92\%), age $>45$ (61\%; 95\% CI: 56-67\%), and were detected through imaging (35\%; 95\% CI: 26-45\%), of which ultrasound was the most common modality (27\%; $95 \%$ CI: $14-43 \%)$. The heterogeneity for all the effect sizes was large and significant.

Conclusions About half of thyroid cancers were found incidentally through the use of imaging studies, in particular neck ultrasound. These incidentally found cancers were mostly small papillary thyroid cancer. These results highlight opportunities for interventions aimed at reducing drivers of overdiagnosis.
\end{abstract}

Keywords Thyroid cancer $\cdot$ Mechanism of detection $\cdot$ Incidental diagnosis $\cdot$ Systematic review $\cdot$ Meta-analysis

\section{Introduction}

The incidence of thyroid cancer has increased worldwide $[1,2]$. In the United States, for instance, it increased from a

Juan P. Brito

brito.juan@mayo.edu

1 Knowledge and Evaluation Research Unit, Mayo Clinic, Rochester, MN 55905, USA

2 Universidad Central, Medical School, Quito-Ecuador, Quito, Ecuador

3 CaTaLiNA: Cancer de tiroides en Latinoamerica, Quito, Ecuador

4 Department of Otolaryngology-Head and Neck Surgery, School of Medicine, Stanford University, Stanford, CA, USA

5 Mayo Clinic, Division of Endocrinology, Diabetes, Metabolism, and Nutrition, Rochester, MN 55905, USA
4.9 to 14.2 per 100,000 person-years over the last two decades [3], while in South Korea, thyroid cancer increased by more than sevenfold, from 6.3 per 100,000 person year in 1999 to 47.5 per 100,000 population in 2009 .

6 Endocrinology Department, Hospital de Especialidades Eugenio Espejo, Quito, Ecuador

7 Surgery Department, Hospital de Especialidades Eugenio Espejo, Quito, Ecuador

8 Universidad San Francisco de Quito, Quito, Ecuador

9 Miami Cancer Institute, Miami, FL, USA

10 Division of Endocrine Surgery, Mayo Clinic, Rochester, MN, USA

11 Unidad de Conocimiento y Evidencia, Universidad Peruana Cayetano Heredia, Lima, Peru 
This difference in thyroid cancer trends reflects underlying different mechanisms of thyroid cancer detection. In South Korea, $90 \%$ of the new thyroid cancers were detected by screening with neck ultrasound. In other countries, however, the mechanism of detection of thyroid cancer is unclear. Outside South Korea, some studies have suggested that the rise of thyroid cancer incidence is driven by the incidental detection of small and asymptomatic thyroid cancer lesions [1, 4-6] by increased use of imaging technologies [7], or detection of thyroid cancer lesions found in the course of histologic review of thyroid glands removed for apparently benign conditions [8, 9]. Others have shown that the increased in incidence is explained by larger, likely palpable and symptomatic, advanced stage thyroid cancer [10].

Understanding the method of thyroid cancer detection has potential implications on interpreting incidence rates, the diagnosis, and management of thyroid cancer. The aim of this study is to summarize the available evidence to assess the frequency of incidental thyroid cancer and examine the triggers that lead to diagnosis with comparison across multiple cohorts internationally.

\section{Methods}

A protocol was developed to perform this study and is available in Online Resource [11]. Overall, this manuscript is reported according to the Preferred Reporting Items for Systematic Reviews and Meta-analysis (PRISMA) guidelines (Fig. 1) [12].

\section{Eligibility criteria and study selection}

We included original studies with patients older than 18 years with thyroid cancer confirmed by histology and reporting non-incidental detected thyroid cancer and at least one incidental pathway of thyroid cancer detection (Fig. 2). Only manuscripts written in English, Spanish, or Portuguese were included. We excluded studies in which the aim was only to assess the impact of thyroid cancer screening (e.g., thyroid cancer screening programs in South Korea).

\section{Data sources and searches}

We applied a search strategy developed in collaboration with an experienced librarian to find potentially eligible studies in Ovid MEDLINE(R) and Epub Ahead of Print, InProcess \& Other Non-Indexed Citations, and Daily, Ovid EMBASE, and Scopus from each database's inception until June, 2020. Controlled vocabulary supplemented with keywords was used to search for studies of triggers of diagnosis in patients with thyroid cancer. The actual strategy is in the Appendix. Conference abstracts, literature reviews, case reports, and editorials were excluded. Reference lists of selected studies were searched to identify additional publications.

\section{Study selection}

Search results were uploaded into a systematic review software program (DistillerSR, Ottawa, ON, Canada) [13]. Reviewers (E.L-N., P.S-P., O.J.P., T.L., B.A-M., T.R.), working independently and in duplicate, screened abstracts and titles and full-text for eligibility using standardized instructions. Before initiating the abstract screening phase, a pilot was performed with 20 articles to assess the clarity of the eligibility criteria among reviewers and modifications were done to refine our instructions accordingly. For abstract screening, articles included by at least one reviewer were considered for full-text screening. Similarly, a pilot was performed with ten articles before starting the full-text screening and no modifications were done. At this stage, only articles included by each pair of reviewers were deemed eligible for this systematic review and in case of disagreements, these were resolved by consensus between the six aforementioned reviewers. Full-text screening agreement was calculated using Cohen's kappa, and it was substantial $(k=0.76)$.

\section{Data collection and management}

For all included articles, one reviewer (E.L-N.) extracted studies data into a spreadsheet form and second reviewer (P.S-P.) checked randomly 30\% of data extracted for accuracy and completeness. No changes were made. Variables extracted were: general characteristics of the studies (author, date of publication, country, study design, data collection period), setting (single center, multicenter and population-based study), participant characteristics (age, sex, mean tumor size, thyroid cancer histological types), and pathways of thyroid cancer diagnosis (incidental and non-incidental diagnosis). Incidental pathway included: (i) cases when a thyroid nodule harboring thyroid cancer was found during the use of imaging test requested for reasons unrelated to a thyroid nodular disorder or symptoms, or (ii) when thyroid cancer was found incidentally in the histological examination of the thyroid gland removed for a benign condition (e.g., goiter, Graves' disease). Non-incidental triggers included cases when a thyroid nodule harboring thyroid cancer was found because a clinician or patient noted an abnormality on physical examination possibly related to the thyroid mass, or patient presented with neck compression symptoms including dysphagia, dysphonia, and neck-pain. 


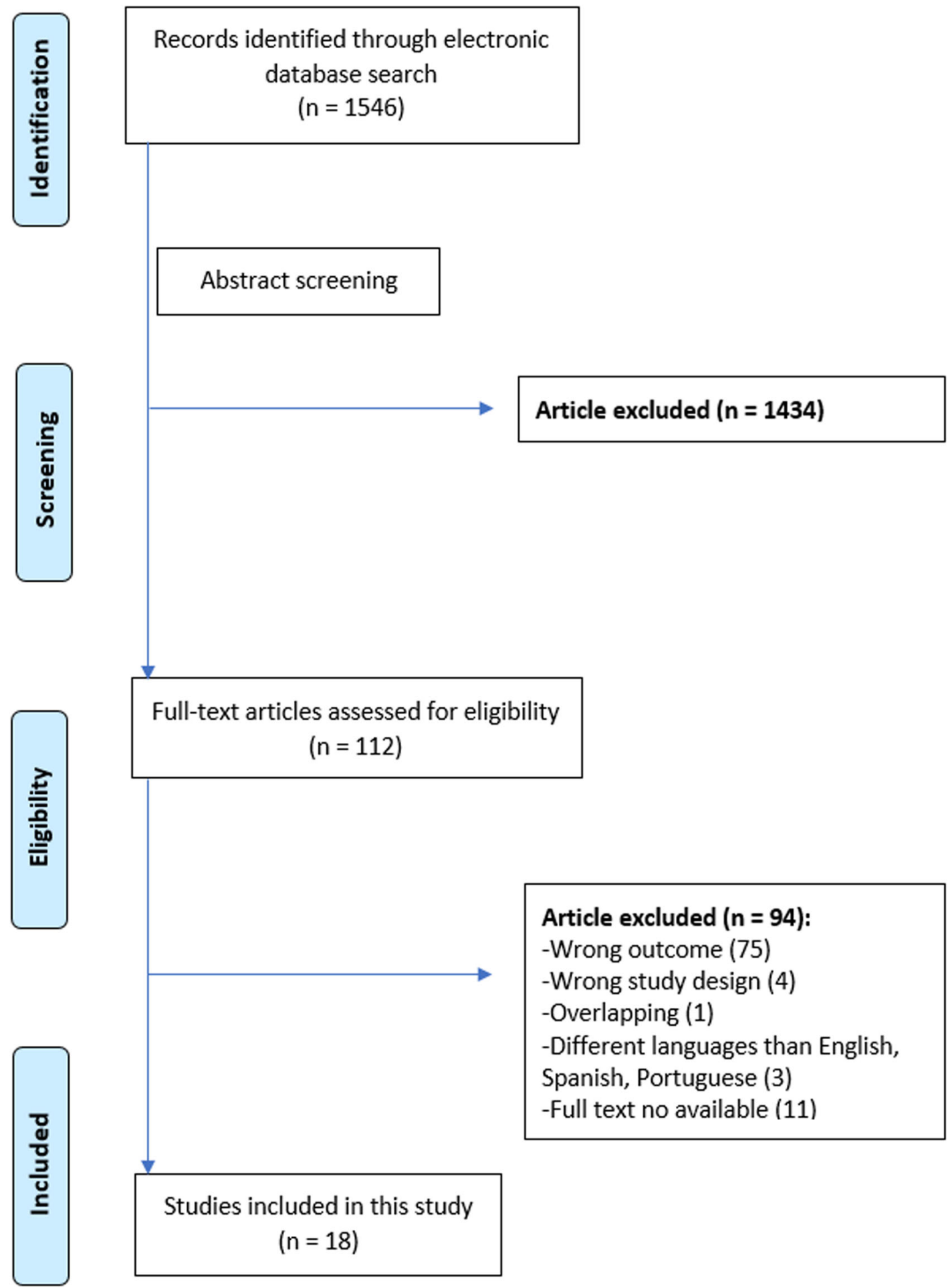

Fig. 1 Preferred Reporting Items for Systematic Reviews and Meta-Analyses (PRISMA) flow diagram of the studies selection process

Additionally, we extracted the type of imaging modality and indications driving the detection of incidental thyroid cancer. Due to variations in the definitions of triggers of thyroid cancer diagnosis, two extractors (E.L-N., P.S-P.) homogenized and discussed the debatable definitions to achieve consensus with the one we proposed. For instance, patients categorized as "non-incidental diagnosis" having thyroid nodules detected by neck ultrasound because of the presence of altered laboratory test results, were categorized to "incidental imaging" [14] 


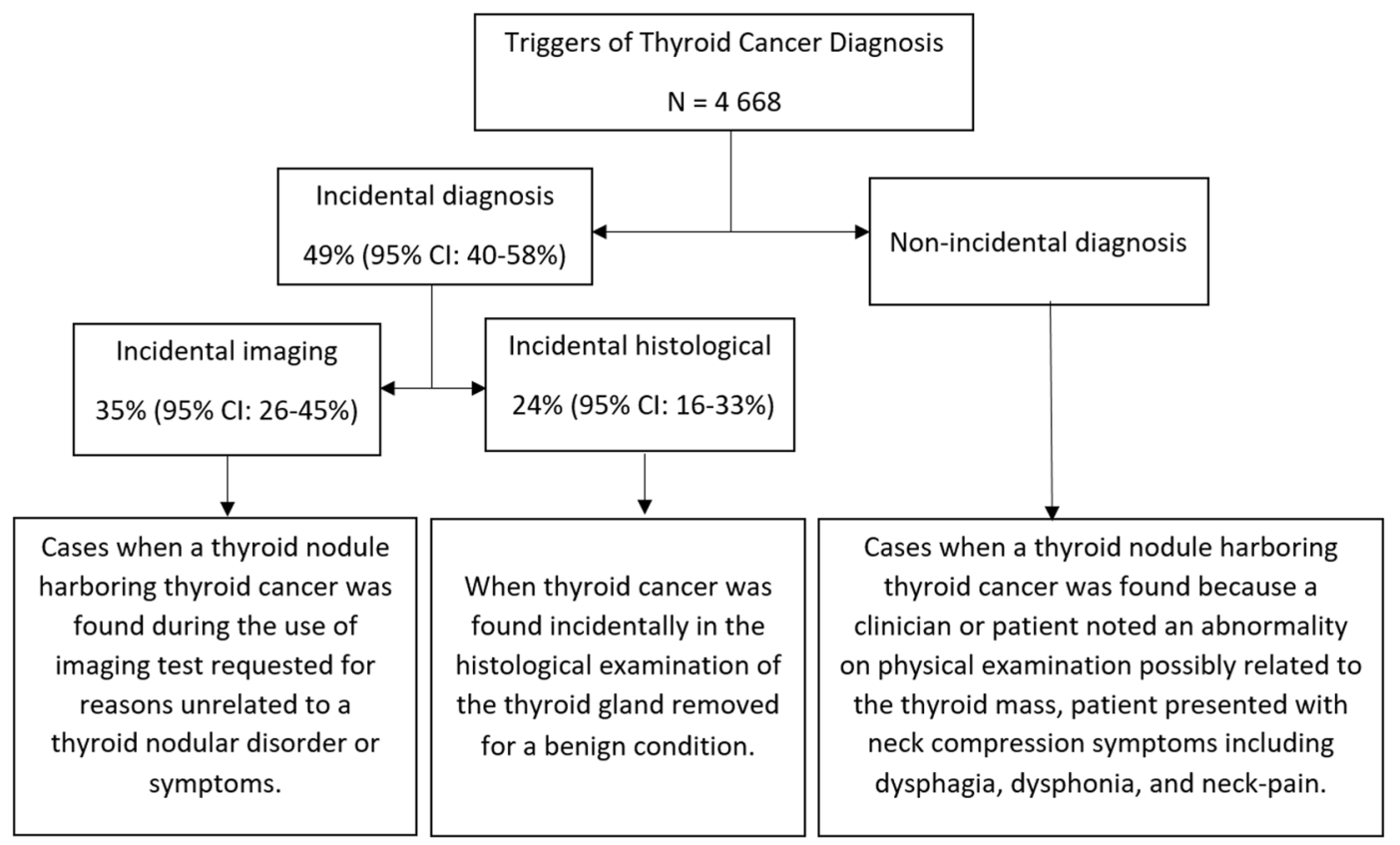

Fig. 2 Definition and frequency of triggers of thyroid cancer diagnosis

(Appendix Table 1). This approach is based on a published framework [9] and recommendations for the use of ultrasound in guidelines [15].

\section{Risk of bias in individual studies}

Study quality was assessed by two independent reviewers (E.L-N. and P.S-P.) based on the nine-star Newcastle Ottawa Scale (NOS) for cohort-studies [16, 17] and an adapted form of the NOS for cross-sectional studies [18], using three predefined domains, namely: a selection of participants (population representativeness), comparability (adjustment for confounders) and ascertainment of outcomes of interests. For both tools, studies that received a score of nine to eight were judged to be at low risk of bias; studies that scored five or seven were considered at moderate risk, and those that scored four or less were considered at high risk of bias.

\section{Statistical analyses}

By using the number of people with an incidental diagnosis and the total number of people in each study, we determined the overall proportion estimates and their confidence intervals $(95 \% \mathrm{CI})$. Every confidence interval was calculated by using the exact method for binomial proportions $[19,20]$ and the overall proportion was estimated with the Freeman-Turkey double arcsine transformation to stabilize the variances [21]. We chose the Freeman-Turkey double arcsine as the main analyses method over others, because it depicts the weights for individual studies and it seems to perform similar to other methods when the proportions are not consistently close to 0 or 1 [22]. The random-effects model was performed with the Dersimonian and Laird method [23]. Since we estimated that we could encounter studies with proportions close to 0 or 1 , a sensitivity analysis was conducted by using generalized linear mixed models [22]. In such scenarios, this meta-analysis model seems to perform better than other (e.g., less biased estimates) [22].

Heterogeneity across studies was assessed with the $I^{2}$ statistic and visually [24]. We considered that $I^{2}<25 \%$ reflected low inconsistency and $I^{2}>75 \%$ reflected high inconsistency. In terms of subgroups analyses, predefined comparisons based on age, sex, and tumor size were planned: $>45$ years vs. $\leq 45$ years, female vs. male, and tumor size $>10 \mathrm{~mm}$ vs. tumor size $\leq 10 \mathrm{~mm}$. Other post-hoc subgroup analyses were additionally executed and they include studies performed in the United States vs. studies performed outside the United States and population-based studies vs. non-population-based studies. The statistical program $\mathrm{R}$ Studio [25] was employed to perform all types of analyses and forest plots.

\section{Online resource}

To support reproducible research, we have made all of our files freely available in an online GitHub platform. Protocol, documents used in the screening phases (references screened in abstract screening and full-text screening), extra 


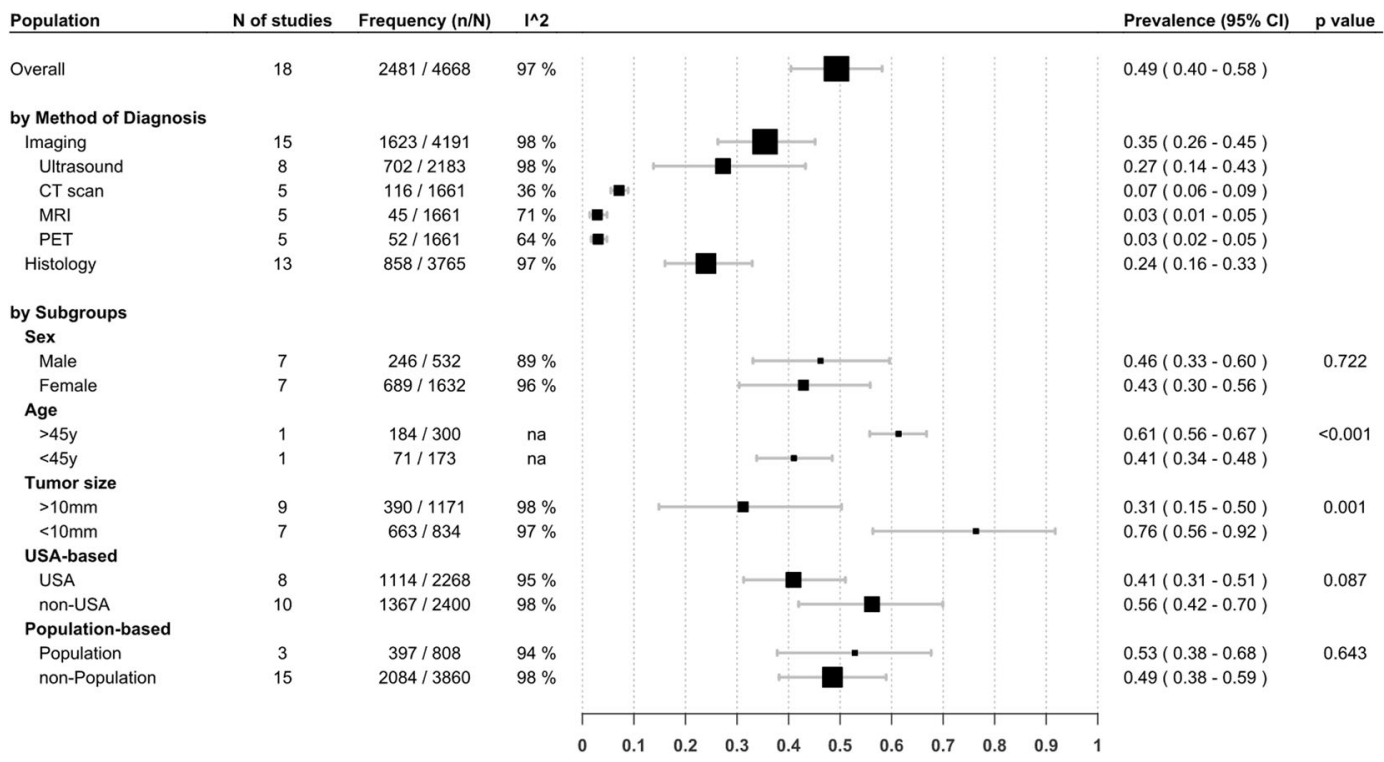

Fig. 3 Overall incidental diagnosis in patients with thyroid cancer by population group

information (tables, figures) and as well as analysis phase (analysis codes or script, raw data) are available in this site (https://github.com/ponceoscarj/Overdiagnosis/blob/master/ Overdiagnosis.md) [11].

\section{Results}

We included 18 studies, conducted between 1991 and 2018, enrolling 4668 patients with thyroid cancer. Figure 2 describes the results of our search. Participants' age ranged from 18 to 89 years old, and most were female $(76 \%)$ with papillary thyroid cancer $(88 \%)$ follicular thyroid cancer $(6 \%)$, and others $(6 \%)$. Around $23 \%$ of all patients with thyroid cancer had papillary thyroid microcarcinoma. Of 18 studies, 1 was cross-sectional [26] and 17 were cohort studies [8-10, 14, 27-39], mostly conducted in the United States [8, 9, 31-35, 39] and Italy [14, 27, 36-38] (Appendix Table 2). Overall, in cohort studies, risk of bias seems to be low (12) to moderate (5), and low in the cross-sectional study (Appendix Table 3).

The overall proportion of people whose thyroid cancer was found incidentally is $49 \%$ [95\% confidence interval (CI): 40-58\%, heterogeneity $\left(I^{2}\right)$ : $97 \%$ ] (Fig. 3).

\section{Overall incidental thyroid cancer diagnosis by triggers of diagnosis}

The proportion of people whose diagnosis of thyroid cancer was triggered by imaging methods was 35\% (95\% CI: $\left.26-45 \%, I^{2}: 98 \%\right)$ and that of triggered by histological examination was $24 \%$ (95\% CI: $16-33 \%, I^{2}: 97 \%$ ). The specific imaging modalities for triggers of incidental thyroid cancer was $27 \%$ (95\% CI: $14-43 \%, I^{2}: 98 \%$ ) for ultrasound, $7 \%$ (95\% CI: 6-9\%, $I^{2}: 36 \%$ ) for computed tomography, $3 \%$ (95\% CI: $2-5 \%, I^{2}: 64 \%$ ) for positron emission tomography, and $3 \%$ (95\% CI: $1-5 \%, I^{2}: 71 \%$ ) for magnetic resonance imaging (Fig. 3).

\section{Overall incidental thyroid cancer diagnosis by subgroups}

Overall incidental thyroid cancer were analyzed by subgroup. Subgroup analysis by tumor size showed that tumor $\leq 10 \mathrm{~mm}$ was incidentally found in $76 \%$ (95\% CI: $\left.56-92 \%, I^{2}: 97 \%\right)$, while tumors $>10 \mathrm{~mm}$ were $31 \%(95 \%$ CI: $15-50 \%, I^{2}: 98 \%$ ), with a $p$ value for interaction test of $<0.001$. Furthermore, the frequency of incidental thyroid cancer was similar in women and men, $43 \%(95 \% \mathrm{CI}$ : $30-56 \%, I^{2}: 96 \%$ ) vs. $46 \%$ (95\% CI: $33-60 \%, I^{2}: 89 \%$ ), respectively, with a $p$ value for subgroup interaction of 0.722 , and different in age $\leq 45$ years $41 \%$ (95\% CI: $34-48 \%$ ) vs. $>45$ years $61 \%$ (95\% CI: $56-67 \%$ ), $p$ value for interaction test of $<0.001$. Similar findings were found when comparing studies performed in the United States (41\%; 95\% CI: $\left.31-51 \%, I^{2}: 95 \%\right)$ vs. studies performed outside the United States (56\%; 95\% CI: 42-70\%, $\left.I^{2}: 98 \%\right)$ with a $p$ value for subgroup interaction of 0.087 , and population-based studies (53\%; 95\% CI: 38-68\%, $\left.I^{2}: 94 \%\right)$ vs. non-population-based studies (49\%; 95\% CI: $38-59 \%, I^{2}: 98 \%$ ) with a $p$ value of 0.643 for interaction (Fig. 3). 


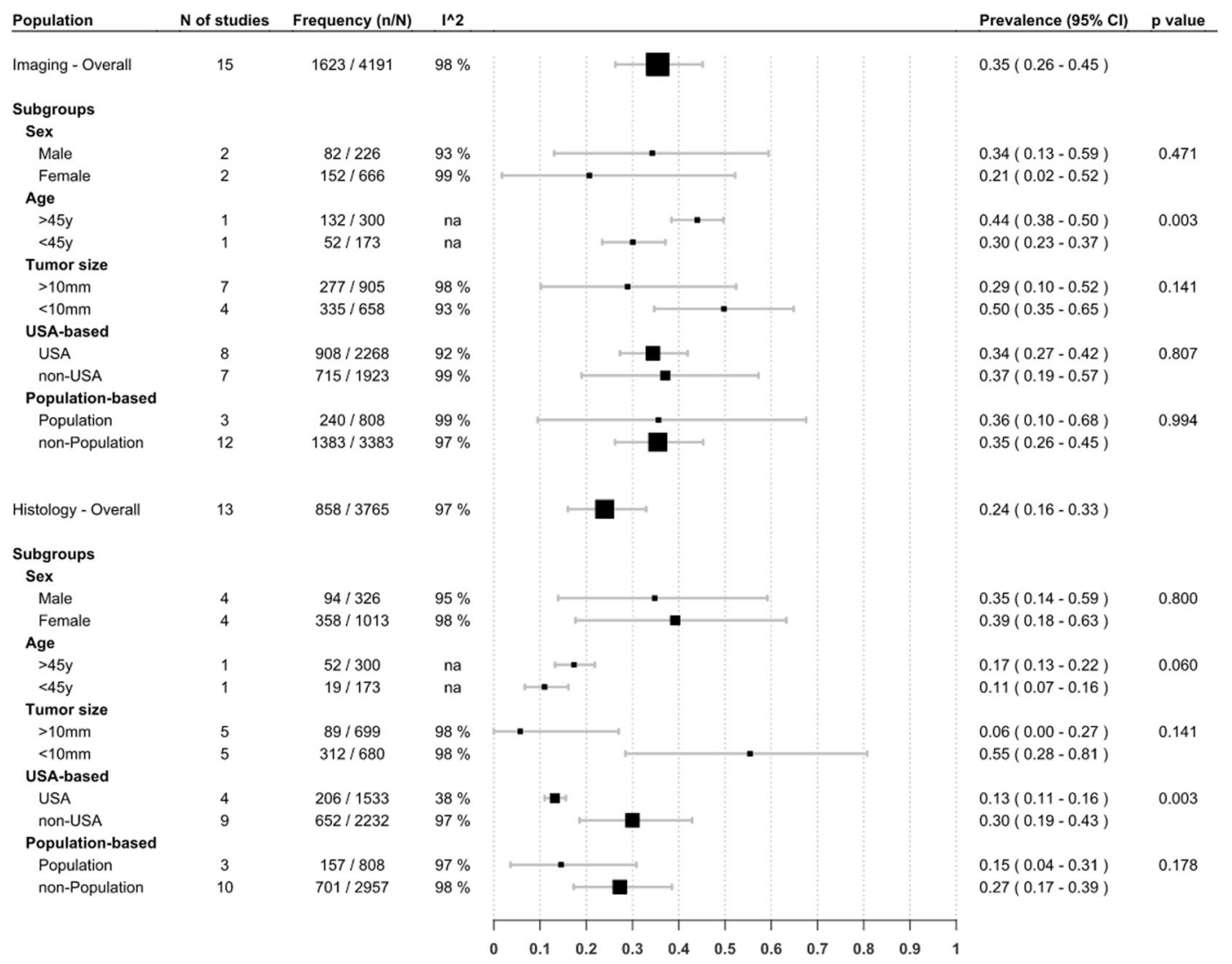

Fig. 4 Imaging and histological incidental diagnosis in patients with thyroid cancer by population group

\section{Incidental imaging by subgroups}

Incidental imaging subgroup analysis by tumor size showed that tumors $\leq 10 \mathrm{~mm}$ were incidentally found in $50 \%$ (95\% CI: $35-65 \%, I^{2}: 93 \%$ ), while tumors $>10 \mathrm{~mm}$ were $29 \%$ (95\% CI: $10-52 \%, I^{2}: 98 \%$ ), with a $p$ value for interaction test of 0.141 . The frequency of incidental imaging was slightly higher in men than women, 34\% (95\% CI: $13-59 \%, I^{2}: 93 \%$ ) vs. $21 \%$ (95\% CI: $02-52 \%$, $\left.I^{2}: 99 \%\right)$, respectively, with a $p$ value for subgroup interaction of 0.471 . These frequencies were similar when comparing studies performed in the United States (34\%; 95\% CI: $27-42 \%, I^{2}$ : 92\%) vs. studies performed outside the United States (37\%; 95\% CI: $19-57 \%, I^{2}$ : $99 \%$ ) with a $p$ value for subgroup interaction of 0.807 , and population-based studies (36\%; $95 \%$ CI: $10-68 \%, I^{2}$ : $99 \%)$ vs. non-population-based studies $(35 \% ; 95 \% \mathrm{CI}$ : $\left.26-45 \%, I^{2}: 97 \%\right)$ with a $p$ value of 0.994 for interaction (Fig. 4).

\section{Incidental histological by subgroups}

Incidental histological subgroup analysis by tumor size showed that tumors $\leq 10 \mathrm{~mm}$ were incidentally found in $55 \%$ (95\% CI: $28-81 \%, I^{2}: 98 \%$ ), while tumors $>10 \mathrm{~mm}$ were $6 \%$ (95\% CI: $\left.0-27 \%, I^{2}: 98 \%\right)$, with a $p$ value for interaction test of 0.141 . Furthermore, the frequency of incidental thyroid cancer was similar in men and women, $35 \%$ (95\% CI: $\left.14-59 \%, I^{2}: 95 \%\right)$ vs. $39 \%$ (95\% CI: $\left.18-63 \%, I^{2}: 98 \%\right)$, respectively, with a $p$ value for subgroup interaction of 0.800 , and was different in studies performed in the United States (13\%; 95\% CI: 11-16\%, $\left.I^{2}: 38 \%\right)$ vs. studies performed outside the United States (30\%; 95\% CI: $\left.19-43 \%, I^{2}: 97 \%\right)$ with a $p$ value for subgroup interaction of $<0.003$. Similar findings were found when comparing population-based studies (15\%; 95\% CI: $04-31 \%, I^{2}:$ 97\%) vs. non-population-based studies (27\%; 95\% CI: $\left.17-39 \%, I^{2}: 98 \%\right)$ with a $p$ value of 0.178 for interaction (Fig. 4).

\section{Indications for incidental diagnosis by ultrasound}

Only three studies reported indications for ultrasound imaging [9, 14, 32]. There were 255 cases that reported indications: family history of thyroid disease and/or presence of altered laboratory test results, such as TSH, FT4, TPO antibodies (62\%), palpated nodule or symptoms not associated with nodule harboring thyroid cancer (15\%), nonspecific neck symptoms (4\%), nonspecific constitutional symptoms (2\%), hyperparathyroidism/ 
hypercalcemia (2\%) gynecological exam (1\%), history of radiation exposure $(1 \%)$, goiter $(1 \%)$, routine sonogram $(1 \%)$, self-sonography $(1 \%)$, previous thyroid surgery (no malignancy) (1\%), and unknown (8\%). Although these studies reported the indications, none identified which were ordered appropriately or not (Appendix Table 3).

\section{Sensitivity analyses}

The overall estimates of studies with proportions different from 0 or $100 \%$, including those close to these numbers (e.g., 1-2\%), were similar in both methods: generalized linear mixed model and Freeman-Turkey double arcsine. Nonetheless, in estimates where one of the studies had a proportion of 0 or $100 \%$, the results were divergent. The Freeman-Turkey double arcsine method in the incidental thyroid cancer subgroup analysis by tumor size showed that for tumor $\leq 10 \mathrm{~mm}$ the proportion was $76 \%$ (95\% CI: $56-92 \%, I^{2}: 97 \%$ ), while for tumors $>10 \mathrm{~mm}$ it was $31 \%$ (95\% CI: $15-50 \%, I^{2}: 98 \%$ ). In contrast, by using the generalized linear mixed model, the estimate for the $\leq 10 \mathrm{~mm}$ group was $83 \%$ (95\% CI: $52-95 \%, I^{2}: 98 \%$ ), whereas for the $>10 \mathrm{~mm}$ the proportion was $26 \%$ (95\% CI: $\left.11-50 \%, I^{2}: 98 \%\right)$. (Online Resource).

\section{Discussion}

\section{Summary of evidence}

We found that a significant proportion of thyroid cancer (49\%) was discovered incidentally in asymptomatic people through histological examination and the use of imaging studies, in particular neck ultrasound (27\%). These incidentally found cancers were mostly small papillary thyroid cancer (76\%). This finding is consistent with a recent study demonstrating that the use of thyroid ultrasound has increased at a rate of 20\% per year from 2002 through 2013 among Medicare patients in the United States, associating this with more thyroid cancer diagnosis [40]. Furthermore, we found among the studies that reported indication for ultrasound use, that $62 \%$ were triggered by abnormal thyroid tests.

Prior to the advent of neck sonography in the 1980s, thyroid cancer nodules had to reach a large size prior to detection; however current neck ultrasonography technology has revolutionized diagnosis and management of thyroid cancer detecting lesions as small as $2 \mathrm{~mm}$ [41]. This increased sensitivity coupled with improved reimbursement and access to US has led to increase in use by endocrinologists close to $80 \%$ [42]. The increased use of thyroid ultrasound raises question about whether or not its use is appropriate. Thyroid ultrasound is indicated when there is a palpable thyroid nodule or in thyroid cancer screening of a high-risk population (e.g., head and neck radiation) [15]. It is unlikely that the increased use of neck ultrasound reflects a surge of new thyroid nodules found by palpation as more than half of patients with thyroid cancer are asymptomatic [9]. In fact, a study showed that many of the thyroid cancers found by ultrasound were detected when clinicians erroneously believed that they felt a nodule and consequently ordered an ultrasound. Interestingly this often revealed no nodule in the palpated area but a small suspicious nodule in the contralateral lobe [9]. Besides ordering thyroid ultrasound due to thyroid nodule misdiagnosis, many thyroid ultrasounds are ordered for the wrong indication. Our analysis shows, in the studies that reported reason for thyroid ultrasound use, that approximately two-third of the thyroid ultrasounds were ordered due to thyroid hormone values abnormalities. The Endocrine Society along with the Choosing Wisely Campaign recommend against the use thyroid ultrasound in patients with abnormal thyroid function tests if there is no palpable abnormality of the thyroid gland [43]. This misuse of thyroid ultrasound, coupled with easy access, increased sensitivity, and the noninvasiveness nature of the test, make thyroid ultrasound an important driver of thyroid cancer diagnosis [41, 42, 44].

Another significant driver of thyroid cancer diagnosis in our study was the histological examination of thyroid glands, particularly when removed in the setting of a benign condition. Parallel to the increased incidence of thyroid cancer, there has been a surge in the diagnosis of thyroid nodules, which in turn has led to higher rates of thyroid surgery [45]. Thyroid surgeries are now more likely to involve the removal of the whole gland, rather than a part of the thyroid [46]. Several autopsy studies have demonstrated that about $11 \%$ of thyroid glands harbor small incidental thyroid cancers and interestingly, there is no significant increase in the prevalence of these lesions since 1949 [47]; thus, the higher rates of thyroid surgery, in particular with removal of the whole gland, will uncover this large reservoir of disease. As shown in this study, most of thyroid cancers detected histologically are smaller than $10 \mathrm{~mm}$. Although factors driving the increase in thyroid surgery for benign conditions remain unclear, it is possible that collective awareness of the high incidence of thyroid cancer could play a factor.

\section{Implications for research and practice}

Although efforts to reduce over treatment of these small thyroid cancer lesions with strategies such as active surveillance are being implemented [48], approaches that 
address avenues promoting thyroid cancer overdiagnosis are needed. Firstly, guidelines should provide recommendations on low-yield diagnostic tests such as unnecessary ultrasound with focus on high-value meaningful use. As ultrasound use becomes adopted into training programs, education on the indications and applicability is crucial. In one study, despite the recent uptake in ultrasound use, $38 \%$ of practicing endocrine surgeons who perform it regularly reported no formal training on its use or applicability [49]. Although, ultrasound is an incredibly useful diagnostic tool, its routine use needs to be re-examined in the context of thyroid cancer overdiagnosis.

\section{Limitations and strengths}

Our study has some limitations, such that some observations should be interpreted with caution. The main limitation of this review is that it can only generate an overview of what is happening with the incidental diagnosis, as we could not describe in detail whether or not the neck ultrasound indications were ordered appropriately in each cohort. Studies are needed to explore this gap; the entry-point into the entire thyroid nodule diagnostic pathway. Another related limitation is the retrospective nature of extraction of the initial primary data. Significant heterogeneity among studies may exist in defining incidental and non-incidental cancers. Consequently, patients could have been misclassified into wrong categories. Likewise, the variations in definitions, made some comparison between studies difficult. Moreover, there is a risk of publication and selection bias given the lack of inclusion of conference abstracts or unpublished literature and inclusion of studies written only in English, Spanish, and Portuguese.

Despite these limitations, the strengths of this study remain notable. Firstly, this is the first global perspective meta-analysis showing rates of incidental thyroid cancer across multiple cohorts allowing for comparison and a summative perspective. Secondly, as we excluded cohorts that were identified through screening programs, we offer a unique perspective into the avenues leading to incidental thyroid cancer diagnosis other than screening. Finally, sensitivity analyses comparing two analyses methods showed that results are consistent in almost all estimates, except in the tumor size subgroup analysis $(\leq 10$ vs. $>10 \mathrm{~mm})$ as studies had proportions of 0 or $100 \%$.

\section{Conclusion}

Our study shows that frequency of incidental thyroid cancer is high across numerous global geographic regions. Half of the thyroid cancers, and almost all papillary thyroid microcarcinomas detected are found incidentally, illustrating that incidental thyroid cancer continues to be a large driver of increasing overall thyroid cancer incidence. Subgroup analysis showed that incidental thyroid cancer tends to represent small cancers likely with indolent course and are primarily diagnosed by ultrasound, prompting a debate surrounding ultrasound misuse. Interventions aimed at reducing this source of overdiagnosis and prospective cohort studies assessing the appropriateness of neck ultrasound indications are needed.

\section{Data availability}

Everything is available in https://github.com/ponceoscarj/ Overdiagnosis/blob/master/Overdiagnosis.md.

\section{Code availability}

Everything is available in https://github.com/ponceoscarj/ Overdiagnosis/blob/master/Overdiagnosis.md.

Author contributions All authors whose names appear on the submission. (1) made substantial contributions to the conception or design of the work; or the acquisition, analysis, or interpretation of data; (2) drafted the work or revised it critically for important intellectual content; (3) approved the version to be published; and (4) agree to be accountable for all aspects of the work in ensuring that questions related to the accuracy or integrity of any part of the work are appropriately investigated and resolved.

\section{Compliance with ethical standards}

Conflict of interest The authors declare that they have no conflict of interest.

Publisher's note Springer Nature remains neutral with regard to jurisdictional claims in published maps and institutional affiliations. 
Table 1 Definition of the triggers of thyroid cancer diagnosis in each study

Reference $\quad$ Definition of the triggers of thyroid cancer diagnosis in each study

Reasons for imaging use

Marina et al. [36]

Rothberger et al. [39]

Russo et al. [10]

Iwata et al. [34]

Seifert et al. [30]

Zagzag et al. [31]

Shakil et al. [35]

González-SánchezMigallón et al. [29]

Provenzale et al. [37]

Brito et al. [9]
Incidental imaging:

-Incidental during carotid power-duplex.

-Incidental during neck imaging other than carotid power-duplex.

-Incidental during imaging work up of thyroid disease, including goiter.

Incidental histology: Pathological examination of an expected benign lesion.

Non-incidental: Patients in whom a nodule harboring thyroid cancer was discovered

because of a self-report of a visual finding or a self-palpation of a neck lump or a complain of compressive symptoms.

Incidental imaging:

- Cases detected on any imaging modality that included the neck but was performed for non-thyroid indications.

- Cases detected on an ultrasound examination specifically performed of the thyroid without clinical suspicion or knowledge of a thyroid nodule. Common reasons for these ultrasound examinations include evaluation of abnormal thyroid function tests, unrelated symptoms such as fatigue or weight gain, or as part of a screening program offered at the workplace.

Non-incidental:

Nodules that were found clinically were nodules palpated by the patient or a physician.

Incidental imaging: Unexpected finding of thyroid cancer in an asymptomatic patient during a routine examination, imaging study, or surgical procedure, or a pathological examination of a surgical specimen in a patient with no thyroid mass, no benign thyroid disease, and no family history of thyroid cancer including ultrasounds performed during a multiphase check-up module.

Non-incidental:

-Detection of thyroid cancer by the patient or people outside the healthcare system. This category includes patients with and without symptoms related to the nodule that

harbored thyroid cancer.

- Clinical evaluation with a presumptive diagnosis of thyroid cancer including a physical exam or diagnostic workup of a cervical mass or lymph node detected by a physician belonging to the healthcare system. This category also included findings by a diagnostic cascade for benign thyroid disease ${ }^{\mathrm{a}}$.

Incidental imaging: Nodules within the thyroid gland that are discovered during diagnostic imaging, which is performed for reasons unrelated to the thyroid gland. Non-incidental: Palpable nodule.

Incidental histological: The patients underwent thyroid surgery with an indication of benign thyroid disease, not for suspicion of differentiate thyroid cancer.

Non-incidental:

-Preoperative suspicion of malignancy ${ }^{\mathrm{a}}$.

-Preoperative assessment of unclear dignity ${ }^{\mathrm{a}}$.

Incidental imaging: Included patients for whom further diagnostic workup was indicated because of a nodule that was initially detected on an imaging study. The indications for these imaging studies were unrelated to the thyroid tumor.

Non-incidental: Included those patients in whom further diagnostic studies were initiated because a physician had noted an abnormality on physical examination or because the patient or another nonprofessional had noted a mass in the neck.

Incidental imaging: histologically proven thyroid cancer initially discovered on imaging not intended for evaluation of the thyroid by computed tomography (CT), magnetic resonance imaging (MRI), positron emission tomography (PET), or ultrasonography (US).

Incidental histological: thyroid cancers discovered histologically after thyroidectomy for benign thyroid diseases, such as Graves' disease or multinodular goiter.

Non-incidental: cancer that had been first discovered due to clinical signs or symptoms or imaging done specifically to evaluate thyroid anatomy

Incidental histological: Malignant neoplasms of thyroid origin, not detected clinically or by preoperative imaging tests, and that are evidenced in the pathological study of the surgical piece removed by an a priori benign process (goiter or hyperthyroidism).

Non-incidental: When the diagnosis of thyroid cancer was established based on clinical, ultrasound, and fine needle aspiration cytology.

Incidental histological: identified postoperatively at histological examination of thyroid in patients submitted to surgery for large multinodular goiter with compressive symptoms and/or incidentally detected in the extra-nodular parenchyma of thyroid gland of patients submitted to surgery for nodules with an 'indeterminate' cytological diagnosis and with a final histological diagnosis of benign nodules.

Non-incidental:

-Diagnosed before surgery as small thyroid nodule incidentally detected at thyroid ultrasound and submitted to FNAC because of the presence of suspicious signs at ultrasound ${ }^{\mathrm{a}}$.

-Papillary thyroid cancer larger than $1 \mathrm{~cm}$.

Incidental imaging:

-When a thyroid nodule harboring thyroid cancer is found during an imaging test requested for reasons unrelated to a thyroid disorder or symptom. This category, however, also includes patients who had an imaging test for possible palpable or symptomatic nodule but the nodule harboring thyroid cancer is not related to any of
Not reported

Not reported

Not reported

Not reported

Not reported

Not reported

Not reported

Not reported

Not reported

Incidental imaging by

ultrasound $(n=54)$ :

-Palpated nodule or

symptoms not associated with nodule harboring 
Table 1 (continued)

\begin{tabular}{ll}
\hline Reference & Definition of the triggers of thyroid cancer diagnosis in each study \\
\hline & these symptoms. \\
& $\bullet$ When a thyroid nodule harboring thyroid cancer is found during the work up of non- \\
& nodular thyroid disease (e.g., patient with hyperthyroidism who has a thyroid ultrasound
\end{tabular}

nodular thyroid disease (e.g., patient with hyperthyroidism who has a thyroid ultrasound positive for a nodule).

-When a thyroid nodule harboring thyroid cancer is found during a physical exam

(thyroid palpation) of an asymptomatic patient.

Incidental histological: Thyroid cancer is found incidentally in the histological

examination of the thyroid gland removed for a benign condition (e.g., Graves' disease).

Non-incidental:

-When a thyroid nodule harboring thyroid cancer is found during a physical exam

(thyroid palpation) or imaging study in a symptomatic patient.

Choi et al. [28]

Incidental imaging: The detection of thyroid nodule by imaging performed for

indications unrelated to the thyroid mass.

Non-incidental:

-The patient presents with complaints possibly related to the thyroid mass such as

dysphagia, dysphonia, neck pain, self-detection of a neck mass, or self-requested screening for thyroid cancer.

-The thyroid nodule detected by a clinician during an evaluation for complaints not related to a thyroid mass.

Bahl et al. [8]

Incidental imaging: Incidental cancers were detected on imaging studies performed for other reasons in patients without any clinical symptoms, examination findings, or suspicion for thyroid cancer.

Incidental histological: An incidental cancer on pathology was found on the surgical specimen when surgery was performed for benign disease or resection of another nodule that was subsequently found to be benign.

Non-incidental:

- A clinical cancer was defined as a palpable nodule.

- A clinical cancer was defined as a nodule discovered in the workup of abnormal thyroid function test or in the context of suspicion for thyroid cancer ${ }^{\mathrm{a}}$.

Malone et al. [32]

Incidental imaging: patients in whom the indication for surgery was a suspicious or malignant cytological finding on fine needle aspiration biopsy (FNAB) of a tumor that was initially detected on an imaging study.

Incidental histological: patients in whom incidental cancers were found on pathological study of the surgical specimen that were not related to the indication for thyroidectomy, the indications for surgery included nodules with suspicious cytology that proved to be benign, symptomatic or enlarging nodules with benign cytology, multinodular goiters, and thyrotoxicosis.

Non-incidental: patients in whom the indication for surgery was a suspicious or malignant cytological finding on FNAB and in whom the patient stated that further diagnostic studies were initiated because a physician had noted an abnormality on physical examination or because the patient or another nonprofessional had noted a mass in the neck

Minuto et al. [38]

Incidental histological: Patients who underwent surgery for presumed benign disease and demonstrated at least 1 focus of thyroid cancer on final histology.

Non-incidental: Patients with clinically evident thyroid cancer.

Kahn et al. [26]

Incidental imaging: Tumor incidentally found during diagnostic imaging for another health problem.

Incidental histological: Cancer diagnosed after surgery for a benign thyroid disorder.

Non-incidental:

-Initially presented due to patient's concern about a mass in their neck or obstructive symptom.

-A doctor noticed a lump in the patient's neck of which they were unaware.

Davies et al. [33]

-The patient specifically requested a thyroid test or evaluation.

-When the identification of a thyroid finding was through a radiologic test done for an indication unrelated to the thyroid gland, such as a computed tomography scan of the

Hypothyroidism $(n=5)$

Unknown $(n=12)$

Not reported

Not reported

Incidental imaging by

ultrasound $(n=98)$ :

-Hashimoto's thyroiditis

$(n=17)$.

-Family history of thyroid

cancer $(n=11)$.

-Hyperthyroidism $(n=$

11).

-Nonspecific neck

symptoms $(n=10)$

-Hypothyroidism $(n=9)$.

-Nonspecific

constitutional symptoms

$(n=6)$.

-Hyperparathyroidism/

hypercalcemia $(n=6)$.

-Gynecological exam ( $n$

$=4$ ).

-History of radiation

exposure $(n=4)$

- Goiter $(n=3)$.

-Previous thyroid surgery

(no malignancy) $(n=2)$.

- "Routine" sonogram ( $n$

$=2$ ).

-Self-sonography $(n=2)$.

- Other lab abnormalities

$(n=2)$.

-Surveillance of another

thyroid nodule $(n=1)$.

-Unknown $(n=8)$

Not reported

Not reported

Not reported 
Table 1 (continued)

chest done for a trauma workup or a chest x-ray done during a preoperative visit. -When the diagnostic cascade stated when a thyroid finding was revealed during a laboratory or other non-radiologic workup for a patient complaint but the thyroid finding did not plausibly explain the patient's presenting complaint.

Non-incidental:

-When a thyroid nodule or other finding had been known about for some time but had been managed expectantly.

-When the person had no complaints referable to the neck or thyroid function and was identified as having a thyroid finding during a routine physical exam performed by a primary care practitioner in a primary care setting.

-A patient was said to be "symptomatic" if the patient's presenting complaint was clearly referable to the region of the thyroid gland or the soft tissues of the neck.

Roti et al. [14]

Incidental histological: Thyroid cancer diagnosed incidentally during thyroid surgery for benign thyroid disorders such as Graves' disease and nodular goiter.

Non-incidental: were thyroid nodules detected by neck ultrasonography conducted for other reasons, because of a family history of thyroid disease and/or presence of altered laboratory test results (TSH, FT4, and thyroid peroxidase antibodies). ${ }^{\mathrm{a}}$

Ruggieri et al. [27]

Incidental histological: Patients in whom the histological diagnosis of cancer was incidental after an operation performed for benign disease.

Non-incidental:

-Patients with a clinically suspicious neoplastic lesion diagnosed before surgical

treatment.

-Patients in whom a neck lymph node metastases were clinically found before diagnosis

of an occult papillary carcinoma in thyroid gland.

${ }^{\text {a }}$ Some non-incidental thyroid cancers where classify as incidental imaging 


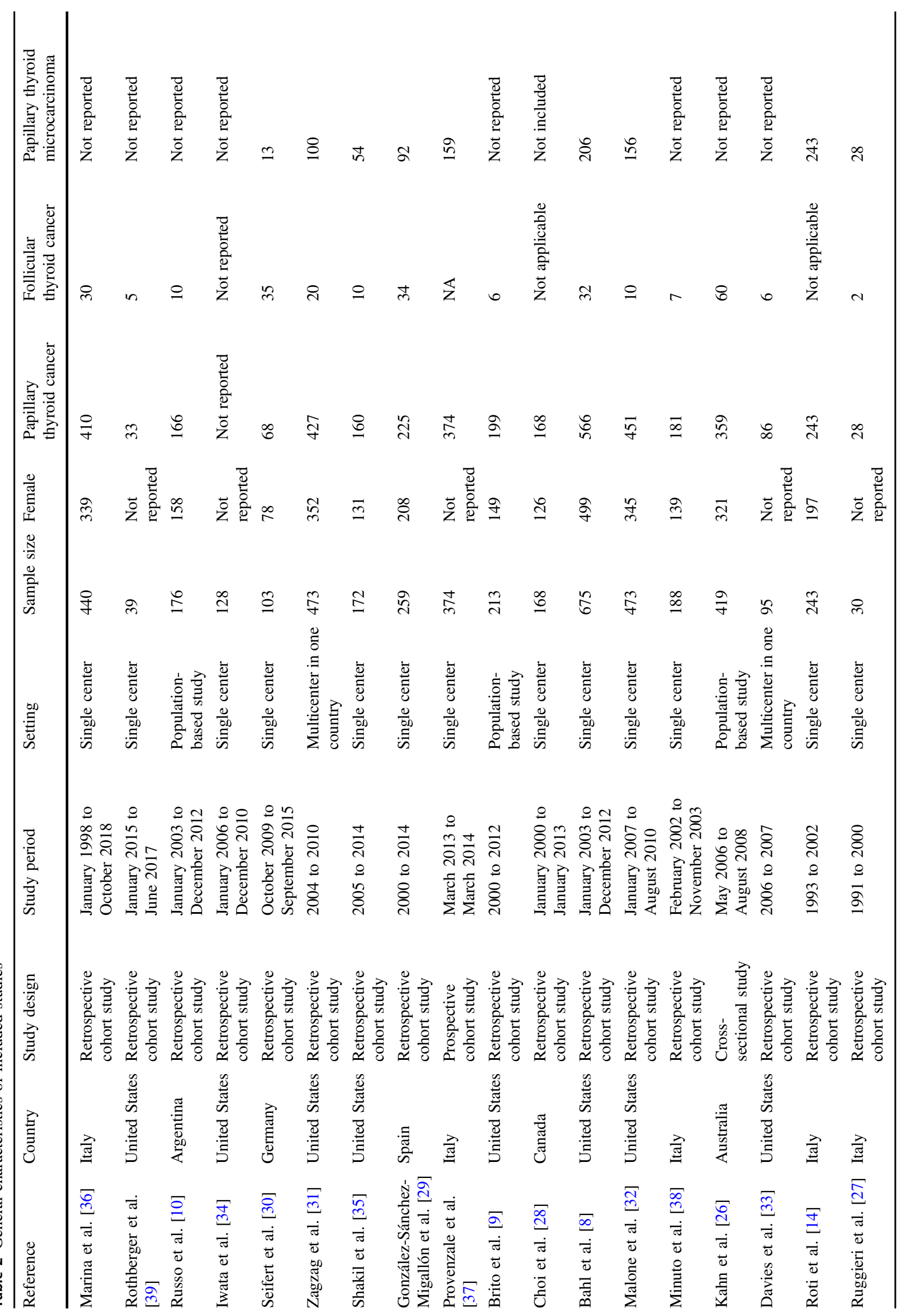




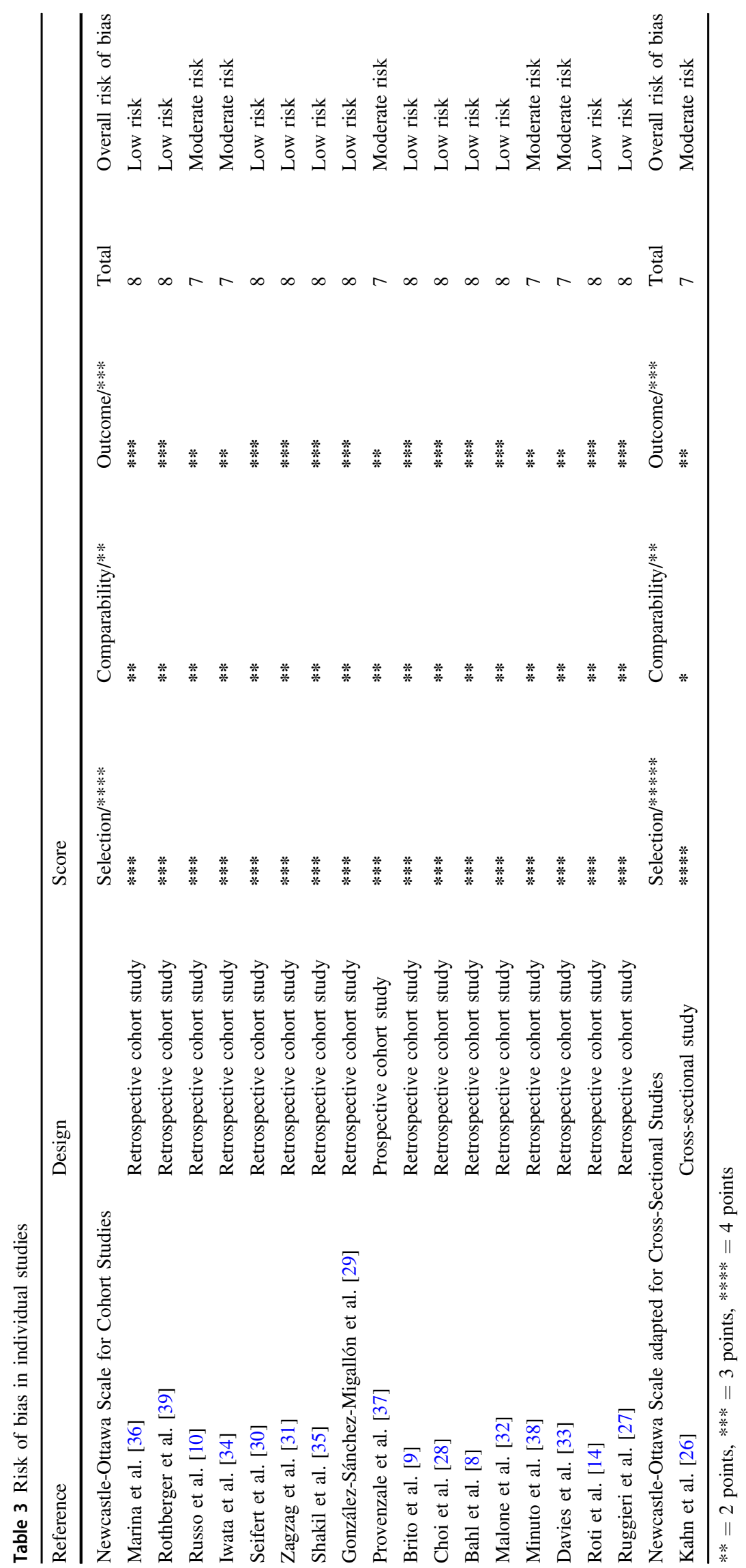




\section{Appendix}

\section{Search strategies}

Ovid

Database(s): Embase 1974 to 2020 June 02, Ovid MEDLINE(R) and Epub Ahead of Print, In-Process \& Other Non-Indexed Citations and Daily 1946 to June 02, 2020 Search Strategy:

\# Searches

Results

1 exp Thyroid Neoplasms/di, dg [Diagnosis, 15,940 Diagnostic Imaging]

2 exp thyroid cancer/di [Diagnosis]

3 (thyroid* adj3 (cancer* or neoplasm* or 139,248 carcinoma*)).ti,ab,hw,kw.

4 (diagnos* or detect* or identify* or identi- $17,002,808$ fied or identification*).ti,ab,hw,kw.

51 or 2 or (thyroid* adj3 (cancer* or 36,818 neoplasm* or carcinoma*) adj3 (diagnos* or detect* or identify* or identified or identification*)).ti,ab,hw,kw.

6 exp incidental findings/

7 exp Mass Screening/

8 exp Palpation/

9 (((diagnos* or detect* or event or events or $2,473,978$ identify* or identified or identification*) adj5 (trigger* or chance)) or incidental or incidentally or palpabl* or palpat* or screen* or serendipit* or subclinical).ti,ab,hw,kw.

106 or 7 or 8 or 9

115 and 10

4847

12 exp comparative study/

13 exp Cohort Studies/

14 exp longitudinal study/

15 exp retrospective study/

16 exp prospective study/

17 exp population research/

18 (cohort* or "longitudinal study" or "longitudinal survey" or "longitudinal analysis" or "longitudinal evaluation" or longitudinal* or ((retrospective or "ex post facto") adj3 (study or survey or analysis or design)) or retrospectiv* or "prospective study" or "prospective survey" or
Table (continued)

"prospective analysis" or prospectiv* or (population adj3 (stud* or survey* or analys* or research)) or "incidence study" or "incidence survey" or "incidence analysis" or (" "follow-up" or followup) adj (stud* or survey or analysis)) or (compar* and (study or trial))).mp,pt.

19 or/12-18

$14,739,985$

2011 and 19

21 limit 20 to (editorial or erratum or note or 8 addresses or autobiography or bibliography or biography or blogs or comment or dictionary or directory or interactive tutorial or interview or lectures or legal cases or legislation or news or newspaper article or overall or patient education handout or periodical index or portraits or published erratum or video-audio media or webcasts) [Limit not valid in Embase,Ovid MEDLINE(R),Ovid MEDLINE(R) Daily Update,Ovid MEDLINE(R) In-Process, Ovid MEDLINE(R) Publisher; records were retained]

2220 not 21

23 remove duplicates from 22

$\underline{\text { Scopus }}$

1. TITLE-ABS-KEY(thyroid* W/3 (cancer* or neoplasm* or carcinoma*) W/3 (diagnos* or detect* or identify* or identified or identification*)).

2. TITLE-ABS-KEY(((diagnos* or detect* or event or events or identify* or identified or identification*) W/ 5 (trigger* or chance)) OR incidental OR incidentally OR palpabl* OR palpat* OR screen* OR serendipit* OR subclinical).

3. TITLE-ABS-KEY(cohort* or "longitudinal study" or "longitudinal survey" or "longitudinal analysis" or "longitudinal evaluation" or longitudinal* or ((retrospective or "ex post facto") W/3 (study or survey or analysis or design)) or retrospectiv* or "prospective study" or "prospective survey" or "prospective analysis" or prospectiv* or (population W/3 (stud* or survey* or analys* or research)) or "incidence study" or "incidence survey" or "incidence analysis" or (("follow-up" or followup) W/1 (stud* or survey or analysis)) or (compar* and (study or trial))).

4. 1 and 2 and 3.

5. DOCTYPE(ed) OR DOCTYPE(bk) OR DOCTYPE(er) OR DOCTYPE(no) OR DOCTYPE(sh). 
6. 4 and not 5 .

7. INDEX(embase) OR INDEX(medline) OR $\operatorname{PMID}\left(0^{*}\right.$ OR $1 *$ OR $2 *$ OR $3 *$ OR $4 *$ OR $5 *$ OR $6 *$ OR $7 *$ OR 8* OR 9*).

8. 6 and not 7 .

\section{References}

1. L. Davies, H.G. Welch, Current thyroid cancer trends in the United States. JAMA Otolaryngol. Head. Neck Surg. 140, 317-322 (2014)

2. B.A. Kilfoy, T. Zheng, T.R. Holford, X. Han, H. Mary, A. Sjodin et al. International patterns and trends in thyroid cancer incidence, 1973-2002. Cancer Causes Control 20, 525-531 (2009)

3. National Cancer Institute. SEER Explorer. Accessed 12 Dec 2019. https://seer.cancer.gov/explorer/application.php?site $=650 \&$ data type $=1 \&$ graph_type $=2 \&$ compareBy $=$ sex $\&$ chk_sex_3 $=3 \&$ chk sex_2 $=2 \& c h k \_r a c e \_1=1 \& c h k \_a g e \_r a n g e \_1=1 \& h d n \_d a t a$ type $=\&$ advopt_precision $=1 \&$ advopt_display $=2 \&$ showDataFor $=$ race_1_and_age_range_1

4. Y. Ito, A. Miyauchi, H. Inoue, M. Fukushima, M. Kihara, T. Higashiyama et al. An observational trial for papillary thyroid microcarcinoma in Japanese patients. World J. Surg. 34, 28-35 (2010)

5. D.T. Hughes, M.R. Haymart, B.S. Miller, P.G. Gauger, G.M. Doherty, The most commonly occurring papillary thyroid cancer in the United States is now a microcarcinoma in a patient older than 45 years. Thyroid 21, 231-236 (2011)

6. L. Davies, L.G.T. Morris, M. Haymart, A.Y. Chen, D. Goldenberg, J. Morris et al. American association of clinical endocrinologists and american college of endocrinology disease state clinical review: the increasing incidence of thyroid cancer. Endocr. Pr. 21, 686-696 (2015)

7. J.P. Brito, J.C. Morris, V.M. Montori, Thyroid cancer: zealous imaging has increased detection and treatment of low risk tumours. BMJ 347, 1-6 (2013).

8. M. Bahl, J.A. Sosa, R.C. Nelson, R.M. Esclamado, K.R. Choudhury, J.K. Hoang, Trends in incidentally identified thyroid cancers over a decade: A retrospective analysis of 2,090 surgical patients. World J. Surg. 38, 1312-1317 (2014)

9. J.P. Brito, A. Al Nofal, V.M. Montori, I.D. Hay, J.C. Morris, The impact of subclinical disease and mechanism of detection on the rise in thyroid cancer incidence: a population-based study in Olmsted county, Minnesota during 1935 through 2012. Thyroid 25, 999-1007 (2015)

10. M.F. Russo Picasso, J. Vicens, C. Giuliani, A.D.V. Jaén, C. Cabezón, M. Figari, et al. Role of the mechanisms of detection in the increased risk of thyroid cancer: a retrospective cohort study in an HMO in Buenos Aires. J. Cancer Epidemiol. 2018, 1-9 (2018)

11. O.J. Ponce, Eddy Lincango-Naranjo. Overdiagnosis. Accessed 28 May 2020. https://github.com/ponceoscarj/Overdiagnosis/blob/ma ster/Overdiagnosis.md

12. A. Liberati, D.G. Altman, J. Tetzlaff, C. Mulrow, P.C. Gøtzsche, J.P.A. Ioannidis et al. The PRISMA statement for reporting systematic reviews and meta-analyses of studies that evaluate health care interventions: explanation and elaboration. PLoS Med. 6, 50931 (2009)

13. S.R. Distiller, Systematic review and literature review software by evidence partners. Accessed 10 Nov 2019. https://www.evidencepa rtners.com/products/distillersr-systematic-review-software/

14. E. Roti, R. Rossi, G. Trasforini, F. Bertelli, M.R. Ambrosio, L. Busutti et al. Clinical and histological characteristics of papillary thyroid microcarcinoma: results of a retrospective study in 243 patients. J. Clin. Endocrinol. Metab. 91, 2171-2178 (2006)

15. B.R. Haugen, E.K. Alexander, K.C. Bible, G.M. Doherty, S.J. Mandel, Y.E. Nikiforov, et al. 2015 American thyroid association management guidelines for adult patients with thyroid nodules and differentiated thyroid cancer: the american thyroid association guidelines task force on thyroid nodules and differentiated thyroid cancer. Thyroid 26, 1-133 (2016). http://online. liebertpub.com/

16. G. Wells, B. Shea, D. O'Connell, J. Peterson, V. Welch, M. Losos, et al. Ottawa Hospital Research Institute. NewcastleOttawa Scale Assess. Qual. nonrandomised Stud. meta-analyses. Accessed 9 Aug 2019. http://www.ohri.ca/programs/clinical_ epidemiology/oxford.asp

17. C. Luchini, B. Stubbs, M. Solmi, N. Veronese, Assessing the quality of studies in meta-analyses: Advantages and limitations of the Newcastle Ottawa Scale. World J. Meta-Anal. 5, 80-84 (2017)

18. R. Herzog, M.J. Álvarez-pasquin, C. Díaz, J. Luis, D. Barrio, J.M. Estrada, et al. Are healthcare workers' intentions to vaccinate related to their knowledge, beliefs and attitudes? A systematic review. BMC Public Health 13, 1-17 (2013)

19. C.J. Clopper, E.S. Pearson, The use of confidence or fiducial limits illustrated in the case of the binomial. Biometrika 26, 404 (1934)

20. S.A. Julious, Two-sided confidence intervals for the single proportion: comparison of seven methods by Robert G. Newcombe, Statistics in Medicine 1998;17:857-872. Stat. Med. 24, 3383-3384 (2005)

21. M.F. Freeman, J.W. Tukey, Transformations Related to the Angular and the Square Root. Ann Math Stat. Inst. Math. Stat. 21, 607-611 (1950)

22. T.A. Trikalinos, P. Trow, C.H. Schmid, Simulation-based comparison of methods for meta-analysis of proportions and rates. Methods Res. Rep. 13, 1-98 (2013). www.effectivehealthcare. ahrq.gov/reports/final.cfm

23. R. DerSimonian, N. Laird, Meta-analysis in clinical trials. Control Clin. Trials 7, 177-188 (1986)

24. J.P.T. Higgins, S.G. Thompson, Quantifying heterogeneity in a meta-analysis. Stat. Med. 21, 1539-1558 (2002)

25. RStudio: Integrated Development for R. RStudio PBM. RStudio tema. Accessed 5 Aug 2020. https://rstudio.com/

26. C. Kahn, L. Simonella, M. Sywak, S. Boyages, O. UNG, D. O'Connell, Pathways to the diagnosis of thyroid cancer in New South Wales: a population-based cross- sectional study. Cancer Causes Control 23, 35-44 (2012)

27. M. Ruggieri, M. Genderini, P. Gargiulo, A. Del Grammastro, A. Mascaro, B. Luongo, et al. Surgical treatment of differentiated microcarcinomas of the thyroid. Eur. Rev. Med Pharm. Sci. 5, 85-89 (2001). http://www.ncbi.nlm.nih.gov/pubmed/ 12004917

28. H. Choi, K. Kasaian, A. Melck, K. Ong, S.J.M. Jones, A. White et al. Papillary thyroid carcinoma: prognostic significance of cancer presentation. Am. J. Surg. 210, 298-301 (2015). https:// doi.org/10.1016/j.amjsurg.2014.12.047

29. E. González-Sánchez-Migallón, B. Flores-Pastor, C.V. PérezGuarinos, J. Miguel-Perelló, A. Chaves-Benito, F. Illán-Gómez et al. Carcinoma de tiroides incidental versus no incidental: presentación clínica, tratamiento quirúrgico y pronóstico. Endocrinol. y. Nutr. 63, 475-481 (2016)

30. P. Seifert, M. Freesmeyer, Preoperative diagnostics in differentiated thyroid carcinoma. NuklearMedizin 56, 201-210 (2017)

31. J. Zagzag, A. Kenigsberg, K.N. Patel, K.S. Heller, J.B. Ogilvie, Thyroid cancer is more likely to be detected incidentally on imaging in private hospital patients. J. Surg. Res. 215, 239-244 (2017). https://doi.org/10.1016/j.jss.2017.03.059 
32. M.K. Malone, J. Zagzag, J.B. Ogilvie, K.N. Patel, K.S. Heller, Thyroid cancers detected by imaging are not necessarily small or early stage. Thyroid 24, 314-318 (2013)

33. L. Davies, M. Ouellette, M. Hunter, H.G. Welch, The increasing incidence of small thyroid cancers: where are the cases coming from? Laryngoscope 120, 2446-2451 (2010)

34. A.J. Iwata, A. Bhan, S. Lahiri, A.M. Williams, A.R. Taylor, S.S. Chang et al. Comparison of incidental versus palpable thyroid nodules presenting for fine-needle aspiration biopsy. Head. Neck 40, 1508-1514 (2018)

35. J. Shakil, M.Z. Ansari, J. Brady, J. Xu, R.J. Robbins, Lower rates of residual/recurrent disease in patients with incidentally discovered thyroid carcinoma. Endocr. Pr. 23, 163-169 (2016)

36. M. Marina, M.F. Serra, R. Aldigeri, G. Ceresini, Incidental versus clinically diagnosed differentiated thyroid cancer in both adult and elderly subjects: histological characteristics and follow-up in a retrospective analysis from a single institution. Endocrine 60, 584-591 (2020)

37. M.A. Provenzale, E. Fiore, C. Ugolini, L. Torregrossa, R. Morganti, E. Molinaro et al. "Incidental" and "non-incidental" thyroid papillary microcarcinomas are two different entities. Eur. J. Endocrinol. 174, 813-820 (2016)

38. M.N. Minuto, M. Miccoli, D. Viola, C. Ugolini, R. Giannini, L. Torregrossa, L. Antonangeli, et al. Incidental versus clinically evident thyroid cancer: a 5-year follow-up study. Head Neck 35, 408-412 (2013)

39. G.D. Rothberger, M. Cohen, P. Sahay, P.T. Szczepanczyk, S. Islam, Method of detection of thyroid nodules: correlation with frequency of fine-needle aspiration and malignancy rate. Head Neck 42, 210-216 (2019)

40. M.R. Haymart, M. Banerjee, D. Reyes-Gastelum, E. Caoili, E.C. Norton, Thyroid ultrasound and the increase in diagnosis of low-risk thyroid cancer. J. Clin. Endocrinol. Metab. 104, 785-792 (2019)

41. E. Marqusee, C.B. Benson, M.C. Frates, P.M. Doubilet, P.R. Larsen, E.S. Cibas et al. Usefulness of ultrasonography in the management of nodular thyroid disease. Ann. Intern. Med. 133, 696-700 (2000)

42. L. Leennhardt, M.O. Bernier, M.H. Boin-Pineau, B. Conte Devolx, R. Maréchaud, P. Niccoli-Sire et al. Advances in diagnostic practices affect thyroid cancer incidence in France. Eur. J. Endocrinol. 150, 133-139 (2004)

43. Endocrine Society-Thyroid ultrasounds I Choosing Wisely. Accessed 20 Aug 2020. https://www.choosingwisely.org/clinicia $\mathrm{n}$-lists/endocrine-society-thyroid-ultrasounds-in-patients-with-a bnormal-thyroid-function-tests/

44. R.S. Groen, J.J. Leow, V. Sadasivam, A.L. Kushner, Review: Indications for ultrasound use in low- and middle-income countries. Trop. Med. Int. Heal. 16, 1525-1535 (2011)

45. N.S. Ospina, S. Maraka, A.E. Espinosa de Ycaza, H.S. Ahn, M.R. Castro, J.C. Morris et al. Physical exam in asymptomatic people drivers the detection of thyroid nodules undergoing ultrasound guided fine needle aspiration biopsy. Endocrine 54, 433-439 (2016)

46. J.A. Sosa, J.W. Hanna, K.A. Robinson, R.B. Lanman, Increases in thyroid nodule fine-needle aspirations, operations, and diagnoses of thyroid cancer in the United States. Surgery 154, 1420-1427 (2013). https://doi.org/10.1016/j.surg.2013.07. 006

47. L. Furuya-Kanamori, K.J.L. Bell, J. Clark, P. Glasziou, S.A.R. Doi, Prevalence of differentiated thyroid cancer in autopsy studies over six decades: a meta-analysis. J. Clin. Oncol. 34, 3672-3679 (2016)

48. M. Haymart, D. Miller, S. Hawley, Active surveillance for lowrisk cancers - a viable solution to overtreatment? N. Engl. J. Med. 377, 203-206 (2017)

49. B.S. Miller, P.G. Gauger, J.T. Broome, R.E. Burney, G.M. Doherty, An international perspective on ultrasound training and use for thyroid and parathyroid disease. World J. Surg. 34, $1157-1163$ (2010) 